Canadian

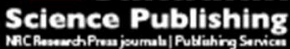

Applied Physiology, Nutrition, and Metabolism Physiologie appliquée, nutrition et métabolisme

\title{
Acute effects of exercise intensity on subsequent substrate utilisation, appetite and energy balance in men and women.
}

\begin{tabular}{|r|l|}
\hline Journal: & Applied Physiology, Nutrition, and Metabolism \\
\hline Manuscript ID & apnm-2017-0280.R2 \\
\hline Manuscript Type: & Article \\
\hline Date Submitted by the Author: & 21 -Jul-2017 \\
\hline Complete List of Authors: & $\begin{array}{l}\text { Shamlan, Ghalia; University of Surrey } \\
\text { Bech, Paul; Imperial College London, Department of Diabetes, } \\
\text { Endocrinology and Metabolism } \\
\text { Robertson, M. Denise; University of Surrey } \\
\text { Collins, Adam; University of Surrey, Nutritional Sciences }\end{array}$ \\
\hline Is the invited manuscript for \\
consideration in a Special \\
Issue? :
\end{tabular}


Acute effects of exercise intensity on subsequent substrate utilisation, appetite and energy balance in men and women.

\section{Ghalia Shamlan ${ }^{1}$, Paul Bech $^{2}$, M Denise Robertson ${ }^{1}$, Adam L Collins $^{1 *}$}

1. Nutritional Sciences, Faculty of Health \& Medical Sciences, University of Surrey, Guildford, Surrey GU2 7XH, UK

2. Department of Diabetes, Endocrinology and Metabolism, Imperial College London, Hammersmith Hospital, London W12 0NN, UK

*Corresponding Author, Dr Adam Collins A.collins@surrey.ac.uk

Tel +44 1483686465

Word count 2998 words (excluding citations) 


\section{Abstract}

Exercise is capable of influencing the regulation of energy balance by acutely modulating appetite and energy intake coupled to effects on substrate utilization. Yet, few studies have examined acute effects of exercise intensity on aspects of both energy intake and energy metabolism, independently of energy cost of exercise. Furthermore, little is known as to the gender differences of these effect. One hour after a standardised breakfast, 40 (19 female), healthy participants (BMI 23.6 $\pm 3.6 \mathrm{~kg} \cdot \mathrm{m}^{-2}$, $\mathrm{VO}_{2 \text { peak }} 34.4 \pm 6.8 \mathrm{ml} \cdot \mathrm{min}^{-1} \cdot \mathrm{min}^{-1}$ ) undertook either High intensity intermittent cycling consisting of 8 repeated 60 s bouts of cycling at $95 \% \mathrm{VO}_{2 \text { peak }}(\mathrm{HIIC})$ or low intensity continuous cycling, equivalent to $50 \% \mathrm{VO}_{2 \text { peak }}(\mathrm{LICC})$, matched for energy cost ( $\left.950 \mathrm{~kJ}\right)$ followed by 90 mins of rest, in a randomised crossover design. Throughout each study visit satiety was assessed subjectively using visual analogue scales alongside blood metabolites and GLP-1. Energy expenditure and substrate utilization were measured over 75 minutes post-exercise via indirect calorimetry. Energy intake was assessed for 48hours post-intervention. No differences in appetite, GLP-1 or energy intakes were observed between HIIC and LICC, with or without stratifying for gender. Significant differences in post exercise non-esterified fatty acid (NEFA) concentrations were observed between intensities in both genders, coupled to a significantly lower respiratory exchange ratio (RER) following HIIC ( $P=0.0028)$, with a trend towards greater reductions in $R E R$ in men $(P=0.079)$. In conclusion, high intensity exercise, if energy matched, does not lead to greater appetite or energy intake but may exert additional beneficial metabolic effects that may be more pronounced in males.

Keywords: Dietary intake, exercise intensity, fat metabolism, gender, energy balance. 


\section{Introduction.}

Exercise and physical activity have long been considered an important factor in the regulation of energy balance and control of body weight (Grundy et al. 1999). Most guidelines on exercise prescribe low/moderate intensity continuous exercise, as a universally acceptable mode for creating a negative energy balance and the prevention or treatment of obesity (Garber et al. 2011). Yet within societies with a high obesity prevalence, the compliance to these guidelines is often poor with a "lack of time" commonly touted as a barrier (Trost et al. 2002). When advocating exercise it is also important to appreciate not simply the responses during the exercise itself but the beneficial postexercise effects (Borsheim \& Bahr 2003, Warren et al. 2009), which may be more pronounced following higher intensity exercise. High intensity intermittent exercise (high intensity work interspersed with low intensity work) is a commonly adopted, acceptable form of high intensity exercise that has been advocated as an effective exercise training method for weight management due to its greater capacity to increase fat oxidation post-exercise (Whyte et al. 2010, Boucher 2011).

Exercise can also influence energy balance by acutely modulating appetite and energy intake, for example via changes in episodic peptides, some of which may be involved in the regulation of metabolic fuels, for example. GLP-1 involvement in insulin secretion (Blundell \& King 1999), and this has been shown experimentally in several studies (Martins et al. 2007, Martins et al. 2008, Martins et al. 2015). In this regard the intensity of exercise is equally important, with more pronounced appetite inhibition shown following higher intensity exercise (Erdmann et al. 2007, Broom et al. 2007). It has been hypothesised that high intensity intermittent exercise may lead to a greater suppression of energy intake and hence an acute negative energy balance (Blundell et al. 2015, Sim et al. 2015), although this may be explained by a greater exercise induced energy deficit.

Quantifying the independent effect of exercise intensity requires a matching of the energy cost of exercise, which is a limitation in some studies (Deighton et al. 2013). Whilst there is some suggestion that appetite changes are no different in response to isoenergetic exercise bouts of 
differing intensity, other metabolic features (such as changes in energy, substrate utilisation, and blood metabolites) may still be distinguishable and could highlight potential advantages of one type of exercise over another. Nevertheless, few studies have examined acutely both the energetic and metabolic effects of exercise intensity.

Gender is another key factor to consider when fully examining exercise and energy balance (Jan Bilski et al. 2009, Hagabian et al. 2013). Gender differences in the metabolic response to exercise are known to exist both during (Devries 2016, Trapp et al. 2007) and post-exercise (Henderson \& Alderman 2014). In order to fully elucidate the best exercise strategy it is important to allow for gender differences, yet, the majority of acute studies on energy balance have been limited to a single genders or are of insufficient cohort size to explore gender differences directly. The novelty of this study is the use of a protocol where the energy cost of exercise is matched to a) simultaneously investigate the independent effects of exercise intensity on both regulation of food intake and energy metabolism, and b) explore any gender distinctions in these synergistic effects. 


\section{Methods.}

\section{Participants}

A total of forty healthy participants (nineteen female) aged 18 to 35 years were recruited from the University of Surrey and wider community (see table 1 for participant characteristics). All participants were weight stable over the 3 months preceding the study ( $\leq 2 \mathrm{~kg}$ variation) and had no significant medical history, or current confounding health issues as determined by medical questionnaire and screening blood sample. All participants exercised recreationally no more than 3 times per week (average $\mathrm{VO}_{2 \text { peak }} 34.4 \pm 6.8 \mathrm{ml} \cdot \mathrm{kg}^{-1} \cdot \mathrm{min}^{-1}$ ). Restrained eaters were identified and excluded using the Dutch Eating Behaviour Questionnaire cut off score of >4 (Van Strien et al. 1986). To control for potential influence of the menstrual cycle between visits female participants were taking oral contraceptives. The study obtained favourable opinion from the University of Surrey Ethics Committee (EC/2011/20/FHMS) and was conducted in accordance with the guidelines laid down in the Declaration of Helsinki. A written informed consent was obtained from all participants before enrolling on the study.

Study Design \& protocol

This was a two-way randomized crossover design acute study. Participants acted as their own controls and completed both High intensity intermittent cycling (HIIC) and Low intensity continuous cycling (LICC), 1 week apart in a randomised order. Following a screening visit, when eligibility was assessed and a 12 lead resting electrocardiogram was performed, participants were asked to attend the Surrey Clinical Research Centre on three occasions. Prior to each visit participants were instructed to refrain from exercise, alcohol and caffeine for $\geq 24$ hours and were supplied with a standardised evening meal $(3740 \mathrm{~kJ}, 120.0 \mathrm{~g}$ carbohydrate, $36.4 \mathrm{~g}$ protein, $30.0 \mathrm{~g}$ fat) to be consumed prior to 20:00 hours the evening before each visit. 
Visit 1: In this pre-intervention visit participants attended at $0800 \mathrm{hrs}$. Body weight and composition were estimated by bioimpedance (Tanita MC180A, Tanita Corp.). Resting (fasted) energy expenditure (REE) and fasted substrate utilisation were measured by indirect calorimetry (GEM Nutrition Ltd). $\quad \mathrm{A} \mathrm{VO}_{2 \text { peak }}$ test was then performed on a cycle ergometer (Lode Corival, $\mathrm{NL}$ ) using a ramp protocol of $20 \mathrm{~W}$ per minute utilising the Metalyser 3B system (Cortex Biophysik GmbH). The maximum wattage obtained $\left(\mathrm{W}_{\max }\right)$ was used to determine individualized workload for the HIIC and LIIC intervention.

Visits 2 and 3: A schematic view of visits 2 and 3 can be seen in figure 1. For each intervention visit (HIIC and LICC) participants attended fasted at 08:00hrs as previously. Upon arrival an indwelling cannula was inserted following which a baseline (fasted) sample was collected. Perceptions of appetite (hunger, fullness and prospective food consumption) were also measured at baseline by self-rated and validated (Flint et al. 2000)) visual analogue scales (VAS). A standardised liquid breakfast was then provided (Dunn's River Nourishment drink; 1668kJ, 54g carbohydrate, $20 \mathrm{~g}$ protein, $12 \mathrm{~g}$ fat). Sixty minutes following the consumption of breakfast another blood sample and VAS measure of appetite were collected immediately prior to participants undertaking either an HIIC or LICC exercise bout. Both exercise bouts, including warm up and cool down, took $\leq 30$ minutes to complete.

Upon completion of the exercise participants laid supine and sampling of blood and appetite continued every 30 minutes for 90 minutes post exercise. Whilst supine, energy expenditure and substrate utilization were also measured at 5,45 and 75 minutes post exercise via indirect calorimetry. At 110 minutes post exercise the incumbent cannula was removed and participants were served with an ad libitum pasta meal as used previously by our research group (Bodinham et al. 2010) (9750kJ, 335.6g carbohydrate, $83.4 \mathrm{~g}$ protein, $71.0 \mathrm{~g}$ fat) and asked to eat until comfortably full. Participants were then free to leave but were supplied with a validated food diary and asked to 
record all food and drink consumed for the next 48 hours. The information from these diaries was translated into nutritional composition using DietPlan 6.0 (Forestfield software Ltd, Horsham, UK).

Exercise protocols

Both exercises were performed on the same electronically controlled cycle ergometer (Lode Corival, Groningen, Netherlands). Participants were fitted with a Hans-Rudolph face mask for collection of breath by breath pulmonary gaseous exchange measurements (Metalyzer 3B, Cortex Biophysik $\mathrm{GmbH}$, Leipzig,) used to determine exercise energy expenditure and substrate utilization (see indirect calorimetry). Both HIIC and LICC were designed to have an energy cost of 1000kJ, calculated using MET values for cycle ergometer (Ainsworth et al 2011). LICC exercise consisted of warm-up at a fixed cadence (60-80rpm) increasing up to $50 \%$ over 2 minutes $\mathrm{VO}_{2 \text { peak }}(75-150 \mathrm{~W})$, participants then maintained $50 \% \mathrm{VO}_{2 \text { peak }}$ at the same fixed cadence for 25 minutes. HIIC exercise was based on the previous protocol of Little et al. (2010), consisting of a 3 minute warm up of continuous cycling at a fixed cadence and then completion of eight repeated 60 second bouts of cycling at $95 \% \mathrm{VO}_{2 \text { peak }}$ (150-300W) interspersed with 90 seconds of active rest at 50W. Participants were allowed to increase cadence during the 60s high intensity bouts. Both HIIC and LICC comprised a 3 minute cool down (from 50 down to $0 \mathrm{~W}$ ).

\section{Indirect calorimetry}

Non-exercise energy expenditure and substrate utilization were measured using an open-circuit indirect calorimeter (Europa Gaseous Exchange Monitor, GEM Nutrition UK) based on the ventilated flow-through technique (Nicholson et al. 1996). REE and subsequent respiratory quotient (RQ) were measured from respiratory $\mathrm{VO}_{2}$ and $\mathrm{VCO}_{2}$, over 20 minutes and in accordance with methodological recommendations by Compher et al. (2006). Post-exercise gaseous exchange measurements were 
taken over 10 minutes at 5, 45 and 75 minutes post-exercise. Resting and post-exercise energy expenditure were calculated using the modified Weir equation (Weir 1949). Post-exercise-substrate utilization was inferred from measured respiratory exchange ratio (RER) and changes in circulating metabolites (NEFA and glucose concentrations). Exercise energy expenditure and substrate utilization was calculated by Metasoft 2.1 software (Cortex Biophysik), assuming a $2 \%$ fixed contribution of protein oxidation during exercise.

\section{Blood biochemistry}

Blood analysis was completed on 33 participants (14 males, 19 female), due to spoilage during storage for 7 male samples. Blood samples were collected into potassium EDTA tubes for the analysis of TAG, NEFA, and insulin, and sodium oxalate tubes for glucose analysis. GLP-1 was collected with 200 kallikrein-inhibiting units of aprotinin per $\mathrm{ml}$ of blood. Blood samples were centrifuged for 10 minutes at $3000 \mathrm{rpm}$ and plasma aliquots stored at $-20 \mathrm{oC}$ prior to batch analysis upon completion of the study. Insulin was analysed using an enzyme linked immunosorbent assay (Millipore Corp; intra/inter assay CV of 7 and 9\% respectively). Glucose, Tri acyl glycerol (TAG) and Non-esterified fatty acids (NEFA) were analysed using commercially available kits (instrumentation Laboratory) for the ILAB650 (Instrumentation Laboratory; Intra/inter-assay CV all $<5 \%$ and $<15 \%$ respectively). Total GLP-1 was measured by the Faculty of Medicine, Imperial College London using an in-house radioimmunoassay (RIA) method (Kreymann et al. 1987) with a sensitivity of $2 \mathrm{pmol} / \mathrm{l}$.

\section{Statistical analysis}

Statistical analysis was carried out using IBM SPSS version 22 (SPSS Inc, Chicago, IL) and GraphPad Prism version 6.07 (GraphPad Sotware Inc.). All variables were checked for normality using the Shapiro-Wilk test and data were expressed as mean and standard errors unless otherwise stated. 
Gender differences in baseline characteristics were determined by independent samples t-test.

Differences between interventions for time course data were assessed by repeated measures ANOVA using exercise intervention and time as independent variables. Differences in mean values between HIIC and LICC were assessed using paired sample t-tests, whereas gender differences within each exercise intervention were assessed by independent sample t-test. Differences between genders for time course data (within each HIIC and LICC leg), were assessed using repeated measures ANOVA with time and gender as independent variables. Statistical significance was accepted at the $5 \%$ level. 


\section{Results}

Baseline characteristics and during exercise

Table 1 displays the participant characteristics stratified by gender. No significant differences were seen in age, $\mathrm{BMI}, \mathrm{REE}$ (corrected for FFM), fasted respiratory quotient (RQ) or $\mathrm{VO}_{2 \text { peak }}$ at baseline between the gender groups. There was, however, a statistically significant difference between HIIC and LICC energy expenditure in both the total cohort and when stratified by gender ( $P \leq 0.008)$, with LICC exhibiting a higher measured energy expenditure (mean difference for the cohort $98.9 \pm 109.5$ kJ). Measured respiratory exchange ratio (RER) was significantly higher during HIIC exercise for the whole cohort $(P<0.001)$, indicative of greater preference for carbohydrate oxidation. However, when stratifying for gender, differences in RER between intensities were significant in males only $(P<0.001)$, not females $(P=0.124)$. Furthermore, averaged RER during HIIC was significantly lower in females compared to males $(P<0.001)$

Appetite, GLP-1 and dietary intakes.

Subjective measures of appetite over time from pre breakfast to 90 minutes post exercise can be seen in figures $2(A-C)$. A significant effect of time was noted for all subjective measures of appetite, $(P<0.001)$ but no effect of exercise intensity nor interaction between intensity and time was observed. A trend towards an effect of exercise intensity was observed for feelings of fullness only $(P=0.055)$. Plasma levels of the appetite related hormone GLP-1 over the same period can be seen in figure 2D. A similar significant effect of time was observed $(P<0.001)$ but there was again no effect of exercise intensity on plasma GLP-1 levels $(P=0.230)$ nor interaction between intensity and time $(P>0.05)$. These conclusions from statistical observations for appetite and GLP-1 were the same when stratifying the data by gender. 
Table 2 displays dietary intake data for the 48 hour periods post-exercise, starting from, and including, the ad-libitum test meal. For both the whole cohort and males and females separately no significant differences between HIIC and LICC were observed in the ad libitum test meal intake ( $P \geq 0.400), 0-24$ hour intake $(P \geq 0.375)$ 24-48 hour intake $(P \geq 0.143)$ or the cumulative total intake over 48 hours $(P \geq 0.216)$. Similarly no significant differences in macronutrient intake (fat, carbohydrate or protein) were observed between HIIC and LICC at any point across the 48 hour period $(P \geq 0.170)$. Interestingly, no significant differences in intake were found between genders except for those indicated in table 2.

Blood metabolites, insulin and substrate utilization.

Plasma levels of NEFA and glucose over time are shown in figure $3(A-D)$, stratified by gender. A significant effect of time was found for NEFA and glucose, in both men and women $(P<0.001)$, but a significant effect of exercise intensity was only noted for NEFA ( $P=0.012$ for men and $P=0.013$ for women). However, glucose incremental area under the curve (iAUC) was significantly higher following HIIC compared to LICC in men (116.8 (SEM 20.3) v. 65.3 (SEM 16.5) mmol/min/L), albeit not significant different in females $(P=0.501)$. For Insulin and triacylglycerol $(T A G)$, there was a significant effect of time $(P<0.001)$ but no significant effect of intervention, nor interaction observed in either the whole cohort, or within gender groups $(P \geq 0.304)$. Insulin and TAG data not shown.

Energy expenditure (per kg FFM) and RER following HIIC and LICC stratified by gender are presented in figure 4 ( $A$ and $B)$. Post-exercise there was a significant effect of exercise intensity on both energy expenditure $(P=0.0028)$ and averaged $R E R(P=0.0052)$. Although in absolute terms there was a significant difference between genders in terms of post exercise $E E(P<0.001)$, there was no significant effect of gender on EE when corrected for fat-free mass (Figure 4A). However, there was a trend towards an effect of gender on RER $(P=0.079)$, figure 4B. 


\section{Discussion}

The aim of this study was to simultaneously examine the acute effects of iso-energetic exercise on appetite, energy intake and energy metabolism. Our protocols of LICC and HIIC were aimed to be matched for energy cost, using a duration of steady state $\mathrm{LICC}$ (at $50 \% \mathrm{VO}_{2 \text { peak }}$ ) matched to the calculated energy cost of the HIIC model by Little et al. (2010). Measured exercise energy expenditure was statistically greater for LIIC, albeit, in absolute terms this translated into a difference of only approximately $100 \mathrm{~kJ}$ ( $25 \mathrm{kcal}$ ) between protocols. It is worth considering that measured exercise energy expenditure via $\mathrm{O}_{2}$ consumption may be underestimated if the exercise is anaerobic, as evident by an observed RER $>1$ in our HIIC leg, due to not fully accounting for the oxygen debt (Scott 2005, 2014). It is also plausible that body temperature, circulation and ventilation were still elevated following cessation of measurement (Borsheim \& Bahr 2003), and hence the true oxygen cost of exercise not being fully captured during our measurement period.

The lack of any significant differences between intensities in terms of appetite and post-exercise energy intake is in agreement with current evidence. Of the few previous studies comparing different intensities matched for energy cost, only one has shown a greater decrease in prospective food intake following high intensity exercise (Imbeault et al. 1997), whilst the majority have demonstrated no difference (Deighton et al. 2013, Pomerleau et al. 2004, Panissa et al. 2016, Howe et al. 2014). Absence of differences in GLP-1 post exercise in our study also agree with other studies comparing different intensities (Ueda et al. 2009, 2013), again, even when matched for energy cost (Howe et al. 2014, Deighton et al 2013a). Similarly, studies have also shown levels of other incretins, such as PYY and ghrelin, have not differed between intensities (Deighton et al. 2013, 2013a, Panissa et al. 2016, Howe et al. 2014).

We failed to show any gender differences in post-exercise appetite and energy intake, which reinforces the observation that at any given intensity there are no noticeable differences between 
men and women (Hagobian et al. 2013). Furthermore, GLP-1, insulin and glucose responses were not different between our males and females as reported by similar studies (Hagobian et al. 2013, Burns et al. 2007). In all, our results confirm the suggestion that when energy cost of exercise is matched, energy intake response is the same for both genders, and that for both men and women this may not fully compensate for the induced energy deficit (Burns et al. 2007, Erdmann et al 2009).

Our study aimed to explore not just the impact of exercise intensity on drivers of energy intake, but energetic and metabolic characteristics between intensities and between genders. Glucose iAUC was significantly higher following HIIC versus LICC in our males but not our females. This observation could be a result of our male participants exercising at a slightly higher relative intensity compared to women during the HIIC leg (as reflected by the higher RER during exercise) leading to greater liver glycogenolysis (Gonzalez et al. 2016). Gender differences in insulin sensitivity, however, may also be a contributing factor (Perreault et al. 2004), with observations of women being more insulin sensitive both during exercise (Boisseau et al. 2000) and in the post-exercise period (Perreault et al. 2004).

Rates of appearance ( $\mathrm{Ra}$ ) of NEFA are known to be $2-3 \times$ greater during exercise of low to moderate intensity (de Glisezinski et al. 2009). In our study clear differences in circulating NEFA were noted between HIIC and LICC, which is likely a consequence of differing catecholamine release between intensities, and known to continue into the post exercise period (Trapp et al. 2007). For example, Erdmann et al (2007) showed post- exercise adrenaline was elevated $~ 500 \%$ above baseline following high intensity cycling compared to only $80 \%$ following Low intensity cycling.

Catecholamine release is a potential stimulant of post exercise mitochondrial respiration, and so it is important to view changes in NEFA in the context of potential changes in substrate utilization. Postexercise RER (HIIC and LICC) was significantly lower, and energy expenditure significantly higher post HICC in both our males and females, and may be suggestive of increased fat oxidation. The observed trend towards a greater reduction in RER for our males supports similar gender differences 
observed by Henderson et al (2007). This sexual dimorphism in exercise response has recently been reviewed by Henderson (2014). The shift towards fat oxidation post-exercise can serve to aid resynthesis of glycogen and removal of $\mathrm{H}+$ built up following high intensity exercise (Nevill et al. 1996). This may, however, also highlight potential errors in quantifying substrate utilization from gaseous exchange, specifically $\mathrm{CO} 2$ production may be underestimated due to the equilibration of the bicarbonate pool, and uncertainty as to the extent of lactate oxidation in liver versus lactate retention in muscle (Frayn 1983). Nevertheless, it is suggested that a more pronounced switch towards fat oxidation in males is a product of higher growth hormone levels (Boutcher et al. 2011), changes in lipoprotein lipase activity (Perreault et al. 2004), coupled to differing energy selection in non-muscle tissues and improved mobilisation of adipose tissue (Mulla et al. 2000). This more pronounced, coordinated response to replenish muscle glycogen may be more necessary in men due to their lower precision of metabolic control (Trapp et al. 2007, Henderson et al. 2008).

Put together, the findings from this study support the notion that for both men and women higher intensity of exercise, if energy matched, does not lead to greater appetite or energy intake. However, higher intensity exercise exerts additional beneficial post-exercise metabolic effects, which may be more pronounced in males than females. More studies are warranted to further explore sexual dimorphism in the role of exercise and energy metabolism.

\section{Acknowledgements.}

The authors report no conflicts of interest associated with this manuscript. This work was funded by the Deanship of Scientific Research at King Saud University. The Authors wish to thank Laura Oakley for her assistance in the dietary analysis. 
References

Ainsworth, B.E., Haskell, W.L, Herrmann, S.D, Meckes, N., Bassett, D.R. Jr., Tudor-Locke, C., et al. 2011. Compendium of Physical Activities: a second update of codes and MET values. Med. Sci.

Sports. Exerc. 43(8): 1575-81. doi: 10.1249/MSS.0b013e31821ece12 .

Blundell, J.E., Gibbons, C., Caudwell, P., Finlayson, G., Hopkins, M. 2015. Appetite control and energy balance: impact of exercise. Obes. Rev. 16 Suppl 1: 67-76. doi: 10.1111/obr.12257

Blundell, J.E., King, N.A. 1999. Physical activity and regulation of food intake: current evidence. Med. Sci. Sports. Exerc. 31(11 Suppl): S573-83. PMID: 10593531

Bodinham, C.L., Frost, G.S., Robertson, M.D. 2010. Acute ingestion of resistant starch reduces food intake in healthy adults. Br. J. Nutr. 103(6): 917-22. doi: 10.1017/S0007114509992534

Boisseau, N., Delamarche, P., Rannou, F., Bentue-Ferrer, D., Gratas-Delamarche, A. 2000. Effects of glucose ingestion at the onset of moderate-intensity, prolonged exercise in women as compared to men. Eur. J. Appl. Physiol. 81(1-2): 93-9. doi: 10.1007/PL00013802

Borsheim, E., Bahr, R. 2003. Effect of exercise intensity, duration and mode on post-exercise oxygen consumption. Sports. Med. 33(14): 1037-60.

Boutcher, S.H. 2011. High-intensity intermittent exercise and fat loss. J. Obes. 2011;2011:868305. doi: $10.1155 / 2011 / 868305$

Broom, D.R., Stensel, D.J., Bishop, N.C. 2007. Exercise-induced suppression of acylated ghrelin in humans. J. Appl. Physiol. (1985). 102(6):2165-71. doi: 10.1152/japplphysiol.00759.2006

Burns, S.F., Broom, D.R., Miyashita, M., Mundy, C., Stensel, D.J. 2007. A single session of treadmill running has no effect on plasma total ghrelin concentrations. J. Sports. Sci. 25(6): 635-42. doi:

$10.1080 / 02640410600831856$ 
Compher, C., Frankenfield, D., Keim, N., Roth-Yousey, L. 2006. Best practice methods to apply to measurement of resting metabolic rate in adults: a systematic review. J. Am. Diet. Assoc. 106(6): 881-903. doi: 10.1016/j.jada.2006.02.009

de Glisezinski, I., Larrouy, D., Bajzova, M., Koppo, K., Polak, J., Berlan, M., et al. 2009. Adrenaline but not noradrenaline is a determinant of exercise-induced lipid mobilization in human subcutaneous adipose tissue. J. Physiol. 587(Pt 13): 3393-404. doi: 10.1113/jphysiol.2009.168906

Deighton, K., Barry, R., Connon, C.E., Stensel, D.J. 2013. Appetite, gut hormone and energy intake responses to low volume sprint interval and traditional endurance exercise. Eur. J. Appl. Physiol. 113(5): 1147-56. doi: 10.1007/s00421-012-2535-1

Deighton, K., Karra, E., Batterham, R.L., Stensel, D.J. 2013a. Appetite, energy intake, and PYY3-36 responses to energy-matched continuous exercise and submaximal high-intensity exercise. Appl. Physiol. Nutr. Metab. 38(9): 947-52. doi: 10.1139/apnm-2012-0484

Devries, M.C. 2016. Sex-based differences in endurance exercise muscle metabolism: impact on exercise and nutritional strategies to optimize health and performance in women. Exp. Physiol. 101(2): 243-9. doi: 10.1113/EP085369

Erdmann, J., Tahbaz, R., Lippl, F., Wagenpfeil, S., Schusdziarra, V. 2007. Plasma ghrelin levels during exercise - effects of intensity and duration. Regul. Pept. 143(1-3): 127-35. doi:

10.1016/j.regpep.2007.05.002

Flint, A., Raben, A., Blundell, J.E., Astrup, A. 2000. Reproducibility, power and validity of visual analogue scales in assessment of appetite sensations in single test meal studies. Int. J. Obes. Relat. Metab. Disord. 24(1): 38-48. PMID: 10702749.

Frayn, K.N. 1983. Calculation of substrate oxidation rates in vivo from gaseous exchange. J. Appl. Physiol. Respir. Environ. Exerc. Physiol. 55(2): 628-34. PMID: 6618956 
Garber, C.E., Blissmer, B., Deschenes, M.R., Franklin, B.A., Lamonte, M.J., Lee, I.M., et al. 2011. American College of Sports Medicine position stand. Quantity and quality of exercise for developing and maintaining cardiorespiratory, musculoskeletal, and neuromotor fitness in apparently healthy adults: guidance for prescribing exercise. Med. Sci. Sports. Exerc. 43(7): 1334-59. doi: 10.1249/MSS.0b013e318213fefb Gonzalez, J.T., Fuchs, C.J., Betts, J. A., van Loon, L.J. 2016. Liver glycogen metabolism during and after prolonged endurance-type exercise. Am. J. Physiol. Endocrinol. Metab. 311(3): E543-53. doi: 10.1152/ajpendo.00232.2016

Grundy, S.M., Blackburn, G, Higgins, M., Lauer, R., Perri, M.G., Ryan, D. 1999. Physical activity in the prevention and treatment of obesity and its comorbidities: evidence report of independent panel to assess the role of physical activity in the treatment of obesity and its comorbidities. Med. Sci. Sports. Exerc. 31(11): 1493-500. PMID: 10593519

Hagobian, T.A., Yamashiro, M., Hinkel-Lipsker, J., Streder, K., Evero, N., Hackney, T. 2013. Effects of acute exercise on appetite hormones and ad libitum energy intake in men and women. Appl. Physiol. Nutr. Metab. 38(1): 66-72. doi: 10.1139/apnm-2012-0104

Henderson, G.C. 2014. Sexual dimorphism in the effects of exercise on metabolism of lipids to support resting metabolism. Front. Endocrinol. (Lausanne). 5: 162. doi: 10.3389/fendo.2014.00162 Henderson, G.C., Alderman, B.L. 2014. Determinants of resting lipid oxidation in response to a prior bout of endurance exercise. J. Appl. Physiol. (1985). 116(1): 95-103. doi:

10.1152/japplphysiol.00956.2013

Henderson, G.C., Fattor, J.A., Horning, M.A., Faghihnia, N., Johnson, M.L., Luke-Zeitoun, M. et al. 2008. Glucoregulation is more precise in women than in men during postexercise recovery. Am. J. Clin. Nutr. 87(6): 1686-94. PMID: 18541557. 
Henderson, G.C., Fattor, J.A., Horning, M.A., Faghihnia, N., Johnson, M.L., Mau, T.L. et al. 2007. Lipolysis and fatty acid metabolism in men and women during the postexercise recovery period. J. Physiol. 584(Pt 3): 963-81. doi: 10.1113/jphysiol.2007.137331

Howe, S.M., Hand, T.M., Manore, M.M. 2014. Exercise-trained men and women: role of exercise and diet on appetite and energy intake. Nutrients, 6(11): 4935-60. doi: 10.3390/nu6114935

Imbeault, P., Saint-Pierre, S., Alméras, N., Tremblay, A. 1997. Acute effects of exercise on energy intake and feeding behaviour. Br. J. Nutr. 77(4): 511-21. PMID: 9155502

Kreymann, B., Williams, G., Ghatei, M.A., Bloom, S.R. 1987. Glucagon-like peptide-1 7-36: a physiological incretin in man. Lancet, 2(8571): 1300-4. PMID:2890903.

Little, J.P., Safdar, A., Wilkin, G.P., Tarnopolsky, M.A., Gibala, M.J. 2010. A practical model of lowvolume high-intensity interval training induces mitochondrial biogenesis in human skeletal muscle: potential mechanisms. J. Physiol. 588(Pt 6): 1011-22. doi: 10.1113/jphysiol.2009.181743

Martins, C., Morgan, L.M., Bloom, S.R., Robertson, M.D. 2007. Effects of exercise on gut peptides, energy intake and appetite. J. Endocrinol. 193(2): 251-8. doi: 10.1677/JOE-06-0030

Martins, C., Robertson, M.D., Morgan, L.M. 2008. Effects of exercise and restrained eating behaviour on appetite control. Proc. Nutr. Soc. 67(1): 28-41. doi: 10.1017/S0029665108005995

Martins, C., Stensvold, D., Finlayson, G., Holst, J., Wisloff, U, Kulseng, B. et al. 2015. Effect of moderate- and high-intensity acute exercise on appetite in obese individuals. Med. Sci. Sports. Exerc. 47(1): 40-8. doi: 10.1249/MSS.0000000000000372

Mulla, N.A., Simonsen. L., Bulow, J. 2000. Post-exercise adipose tissue and skeletal muscle lipid metabolism in humans: the effects of exercise intensity. J. Physiol. 524. Pt 3: 919-28. PMID:

10790168 
Nevill, M.E., Holmyard, D.J., Hall, G.M., Allsop, P., van Oosterhout, A., Burrin, J.M. et al. 1996. Growth hormone responses to treadmill sprinting in sprint- and endurance-trained athletes. Eur. J. Appl. Physiol. Occup. Physiol. 72(5-6): 460-7. PMID: 8925817

Nicholson. M.J., Holton, J., Bradley, A.P., Beatty, P.C., Campbell, I.T. 1996. The performance of a variable-flow indirect calorimeter. Physiol. Meas. 17(1): 43-55. doi: Doi 10.1088/0967$3334 / 17 / 1 / 005$

Panissa, V.L., Julio, U.F., Hardt, F., Kurashima, C., Lira, F.S., Takito, M.Y. et al. 2016. Effect of exercise intensity and mode on acute appetite control in men and women. Appl. Physiol. Nutr. Metab. 41 (10): 1083-91. doi: 10.1139/apnm-2016-0172

Perreault, L., Lavely, J.M., Bergman, B.C., Horton, T.J. 2004. Gender differences in insulin action after a single bout of exercise. J. Appl. Physiol. (1985). 97(3): 1013-21. doi:

10.1152/japplphysiol.00186.2004

Perreault, L., Lavely, J.M., Kittelson, J.M., Horton, T.J. 2004. Gender differences in lipoprotein lipase activity after acute exercise. Obes. Res. 12(2): 241-9. doi: 10.1038/oby.2004.31

Pomerleau, M., Imbeault, P., Parker, T., Doucet, E. 2004. Effects of exercise intensity on food intake and appetite in women. Am. J. Clin. Nutr. 80(5): 1230-6. PMID: 15531670.

Scott, C.B. 2005. Misconceptions about Aerobic and Anaerobic Energy Expenditure. J. Int. Soc. Sports. Nutr. 2: 32-7. doi: 10.1186/1550-2783-2-2-32

Scott, C.B. 2014. Combustion, respiration and intermittent exercise: a theoretical perspective on oxygen uptake and energy expenditure. Biology, (Basel). 3(2): 255-63. doi: 10.3390/biology3020255

Sim, A.Y., Wallman, K.E., Fairchild, T.J., Guelfi, K.J. 2015. Effects of High-Intensity Intermittent Exercise Training on Appetite Regulation. Med. Sci. Sports. Exerc. 47(11): 2441-9. doi: 10.1249/MSS.0000000000000687 
Trapp, E.G., Chisholm, D.J., Boutcher, S.H. 2007. Metabolic response of trained and untrained women during high-intensity intermittent cycle exercise. Am. J. Physiol. Regul. Integr. Comp. Physiol. 293(6): R2370-5. doi: 10.1152/ajpregu.00780.2006

Trost, S.G., Owen, N., Bauman, A.E., Sallis, J.F., Brown, W. 2002. Correlates of adults' participation in physical activity: review and update. Med. Sci. Sports. Exerc. 34(12): 1996-2001. doi:

10.1249/01.MSS.0000038974.76900.92

Ueda, S.Y., Miyamoto, T., Nakahara, H., Shishido, T., Usui, T., Katsura, Y. et al. 2013. Effects of exercise training on gut hormone levels after a single bout of exercise in middle-aged Japanese women. Springerplus, 2(1): 83. doi: 10.1186/2193-1801-2-83

Ueda, S.Y., Yoshikawa, T., Katsura, Y., Usui, T., Fujimoto, S. 2009. Comparable effects of moderate intensity exercise on changes in anorectic gut hormone levels and energy intake to high intensity exercise. J. Endocrinol. 203(3): 357-64. doi: 10.1677/JOE-09-0190

Van Strien, T., Frijters, J.E.R., Bergers, G.P.A., et al. 1986. The Dutch Eating Behavior Questionnaire (Debq) for Assessment of Restrained, Emotional, and External Eating Behavior. Int. J. Eat. Disorder. 5(2):295-315. Doi: 10.1002/1098-108x(198602)5:2<295::Aid-Eat2260050209>3.0.Co;2-T

Weir, J.B. 1949. New methods for calculating metabolic rate with special reference to protein metabolism. J. Physiol. 109(1-2): 1-9. PMID: 15394301;

Whyte, L.J., Gill, J.M., Cathcart, A.J. 2010. Effect of 2 weeks of sprint interval training on healthrelated outcomes in sedentary overweight/obese men. Metabolism. 59(10): 1421-8. doi:

10.1016/j.metabol.2010.01.002 
Table 1: Participant characteristics (expressed as mean \pm standard deviation)

\begin{tabular}{|c|c|c|c|}
\hline Age (years) & $23 \pm 4.6$ & $24 \pm 5.3$ & $23 \pm 3.7$ \\
\hline Weight (kg) & $71.5 \pm 15.8$ & $78.9 \pm 13.9$ & $63.4 \pm 14.0 *$ \\
\hline $\mathrm{BMI}\left(\mathrm{kg} / \mathrm{m}^{2}\right)$ & $23.6 \pm 3.6$ & $24.1 \pm 3.4$ & $23.0 \pm 3.8$ \\
\hline $\operatorname{REE}(\mathrm{MJ} /$ day) & $6.22 \pm 0.98$ & $6.72 \pm 0.98$ & $5.68 \pm 0.64 *$ \\
\hline REE/FFM (kJ/kg) & $73.2 \pm 20.6$ & $71.7 \pm 15.5$ & $74.9 \pm 25.5$ \\
\hline Fasted RQ & $0.871 \pm 0.070$ & $0.868 \pm 0.068$ & $0.874 \pm 0.073$ \\
\hline LICC exercise RER & $0.988 \pm 0.038$ & $0.999 \pm 0.043$ & $0.978 \pm 0.029$ \\
\hline HIIC exercise RER & $1.057 \pm 0.084 \dagger$ & $1.112 \pm 0.067 \dagger$ & $0.996 \pm 0.053 *$ \\
\hline
\end{tabular}

$\mathrm{BMI}=$ body mass index, REE = Resting Energy Expenditure, FFM = Fat Free Mass, LICC= low intensity continuous cycling, HIIC = High intensity intermittent cycling. RER = Respiratory Exchange Ratio (averaged breath by breath measure across full duration of exercise)

* Significant difference between genders $(P<0.01)$

† Significant different from LICC $(P<0.01)$ 
Table 2: Adlibitum meal, daily and cumulative energy intakes post LICC and HIIC exercise (expressed as mean \pm standard deviation).

\begin{tabular}{|c|c|c|c|c|}
\hline & \multicolumn{2}{|c|}{ Males $(n=21)$} & \multicolumn{2}{|c|}{ Females $(n=19)$} \\
\hline & LICC & HIIC & LICC & HIIC \\
\hline Ad-libitum meal consumption (MJ) & $5.12 \pm 0.94$ & $5.26 \pm 1.07 *$ & $4.35 \pm 1.72$ & $4.04 \pm 1.25$ \\
\hline 0-24 hour intake (MJ) & $10.37 \pm 5.30$ & $10.01 \pm 4.40$ & $7.79 \pm 3.25$ & $6.86 \pm 3.96$ \\
\hline 24-48 hour intake (MJ) & $10.14 \pm 3.93$ & $8.54 \pm 3.46$ & $7.72 \pm 3.68$ & $7.75 \pm 3.52$ \\
\hline cumulative 48 hour intake (MJ) & $20.51 \pm 5.99 *$ & $18.55 \pm 6.71$ & $15.51 \pm 5.85$ & $14.60 \pm 6.45$ \\
\hline
\end{tabular}

LICC = low intensity continuous cycling, HIIC = High intensity intermittent cycling. ${ }^{*}$ Significantly different from females $(P<0.05)$. No significant differences seen between HIIC and LICC $(P \geq 0.171)$. 
Figure 1: Schematic representation of study visits.

Figure 2: A. Profiles of subjective feelings of fullness $(\mathrm{cm})$ before and after a standardised breakfast (1668kJ), and post LICC ( $\square)$ and HIIC ( $\mathbf{\Delta}$ ) exercise, values represent mean \pm SEM for 40 subjects. Repeated measures ANOVA showed a significant effect of time $(P<0.001)$ but no effect of exercise intervention or interaction. B. Profiles of subjective feelings of hunger $(\mathrm{mm})$ before and after a standardised breakfast (1668kJ), and post LICC ( $\square$ ) and HIIC ( $\mathbf{\Delta}$ ) exercise, values represent mean \pm SEM for 40 subjects. Repeated measures ANOVA showed a significant effect of time $(P<0.001)$ but no effect of exercise intervention or interaction. C. Profiles of subjective feelings of how much they feel they can eat $(\mathrm{mm})$ before and after a standardised breakfast (1668kJ), and post LICC ( $\square$ ) and HIIC ( $\mathbf{\Delta}$ ) exercise, values represent mean \pm SEM for 40 subjects. Repeated measures ANOVA showed a significant effect of time $(P<0.001)$ but no effect of exercise intervention or interaction $D$. GLP-1 plasma level profile ( $\mathrm{pmol} / \mathrm{L}$ ) before and after a standardised breakfast (1668kJ), and post LICC $(\square)$ and HIIC ( $\mathbf{\Delta})$ exercise, values represent mean \pm SEM for 33 subjects. Repeated measures ANOVA showed a significant effect of time $(P<0.001)$ but no effect of exercise intervention or interaction.

Figure 3: A. Plasma NEFA profiles pre and post LICC ( $\square$ ) and HIIC ( $\mathbf{\Delta})$ exercise for males (n=14). Repeated measures ANOVA showed significant effect of time $(P<0.001)$ and exercise intervention $(P=0.012)$, with significant time*exercise interaction $(P<0.001)$. B. Plasma NEFA profiles pre and post LICC $(\square)$ and HIIC ( $\mathbf{A})$ exercise for females ( $n=19)$. Repeated measures ANOVA showed significant effect of time $(P<0.001)$ and exercise intervention $(P=0.013)$, with significant time*exercise interaction $(P<0.001)$. C. Plasma glucose profiles pre and post LICC $(\square)$ and HIIC ( $\mathbf{\Delta})$ exercise for males $(n=21)$. Repeated measures ANOVA showed significant effect of time $(P<0.001)$ but no effect of exercise intervention ( $P=0.142)$, however the iAUC for glucose was significantly greater post-HIIC exercise $(P<0.001)$. C. Plasma glucose profiles pre and post LICC $(\square)$ and HIIC ( $\mathbf{\Delta})$ 
exercise for females $(n=19)$. Repeated measures ANOVA showed significant effect of time $(P<0.001)$ but no effect of exercise intervention ( $P=0.847)$, in addition, iAUC for glucose post-exercise was not significantly different between exercise intensities $(P \geq 0.501)$.

Figure 4 - A - Energy expenditure (EE) via indirect calorimetry, expressed per kg fat-free mass, averaged from measures at 5, 45 and 75 minutes post-exercise in in 21 males $(\bullet)$ and 19 females (घ). Repeated measures ANOVA showed a significant effect of exercise intensity $(P=0.0028)$, but no significant difference between genders or intensity* gender interaction. B- Respiratory exchange ratio (RER) averaged from measures at 5, 45 and 75 minutes post exercise in 21 males (๑) and 19 females ( $\square)$. Repeated measures ANOVA showed a significant effect of intensity $(P=0.005)$, and a trend towards differences between genders $(P=0.079)$, albeit no significant intensity*gender interaction. HIIC = high intensity interval cycling, LICC = low intensity continuous cycling. 


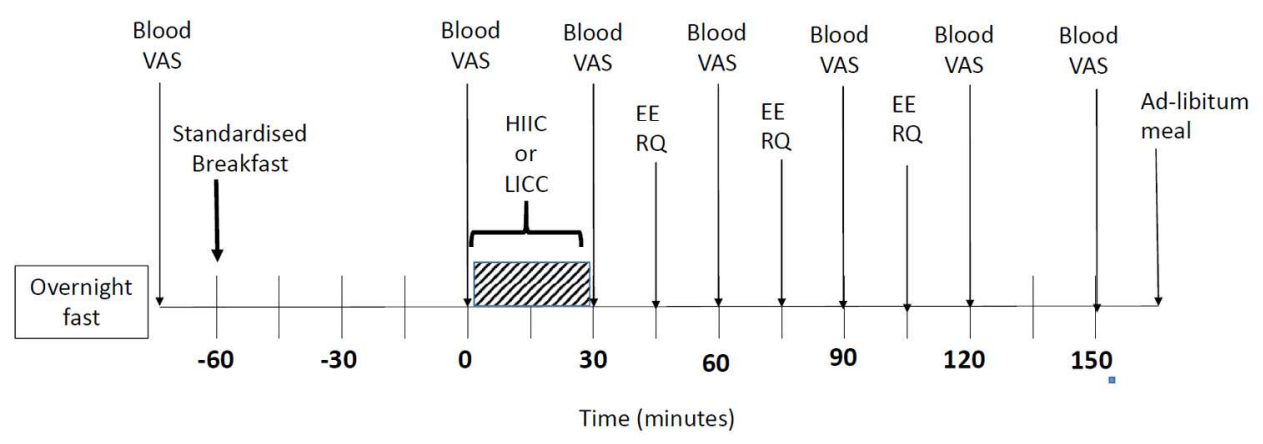

Figure 1: Schematic representation of study visits.

$$
150 \times 73 \mathrm{~mm}(300 \times 300 \mathrm{DPI})
$$


A
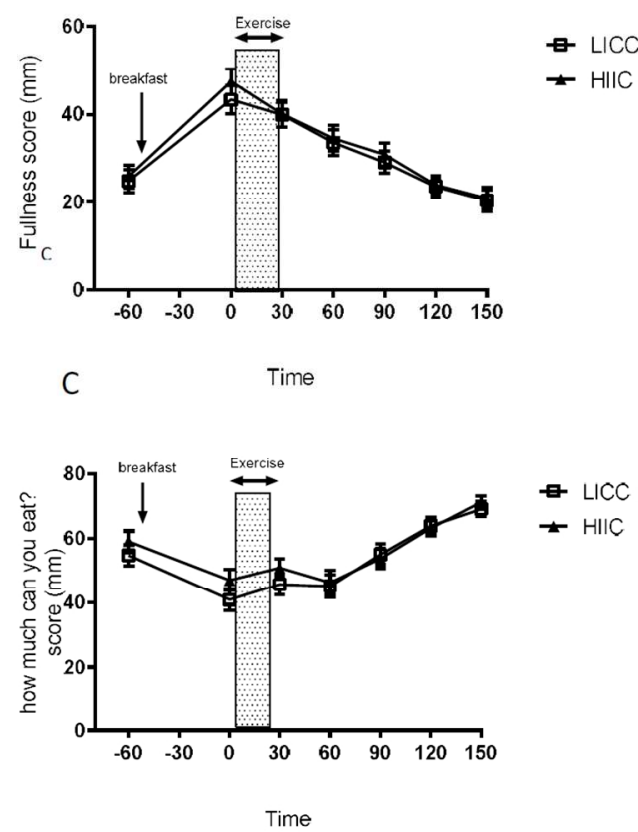

B
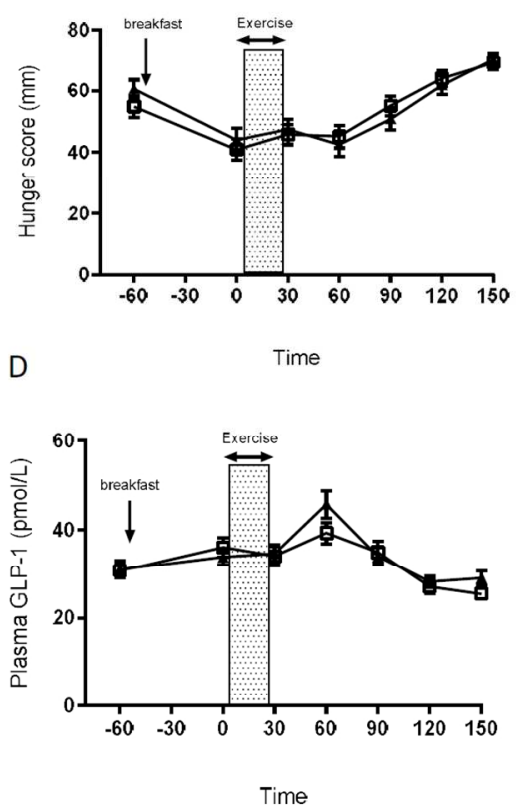

Figure 2: A. Profiles of subjective feelings of fullness $(\mathrm{cm})$ before and after a standardised breakfast (1668kJ), and post LICC $(\leq)$ and HIIC $(\pi)$ exercise, values represent mean \pm SEM for 40 subjects. Repeated measures ANOVA showed a significant effect of time $(P<0.001)$ but no effect of exercise intervention or interaction. B. Profiles of subjective feelings of hunger $(\mathrm{mm})$ before and after a standardised breakfast (1668kJ), and post LICC $(\leq)$ and HIIC $(\pi)$ exercise, values represent mean \pm SEM for 40 subjects. Repeated measures ANOVA showed a significant effect of time $(P<0.001)$ but no effect of exercise intervention or interaction. C. Profiles of subjective feelings of how much they feel they can eat $(\mathrm{mm})$ before and after a standardised breakfast $(1668 \mathrm{~kJ})$, and post LICC $(\leq)$ and HIIC $(\pi)$ exercise, values represent mean \pm SEM for 40 subjects. Repeated measures ANOVA showed a significant effect of time $(P<0.001)$ but no effect of exercise intervention or interaction D. GLP-1 plasma level profile $(\mathrm{pmol} / \mathrm{L})$ before and after a standardised breakfast (1668kJ), and post LICC $(\leq)$ and HIIC $(\pi)$ exercise, values represent mean \pm SEM for 33 subjects.

Repeated measures ANOVA showed a significant effect of time $(P<0.001)$ but no effect of exercise intervention or interaction.

$125 \times 87 \mathrm{~mm}(300 \times 300$ DPI $)$ 
A Males $(n=14)$

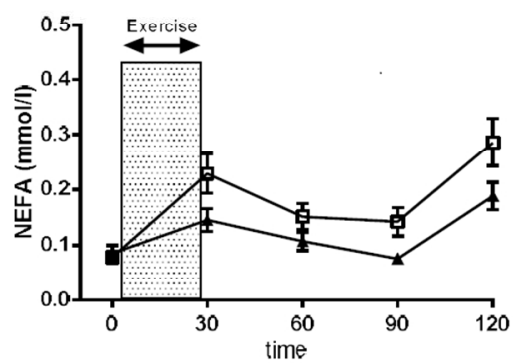

C

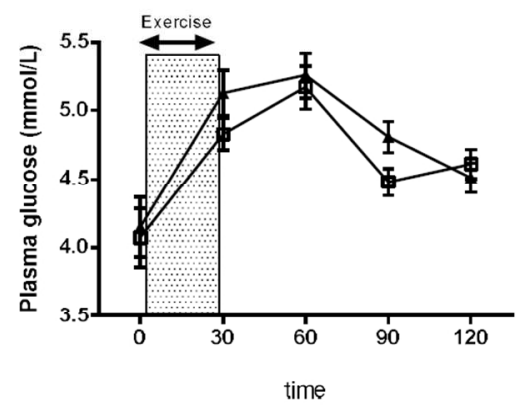

B Females $(n=19)$

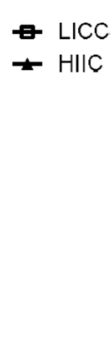

D

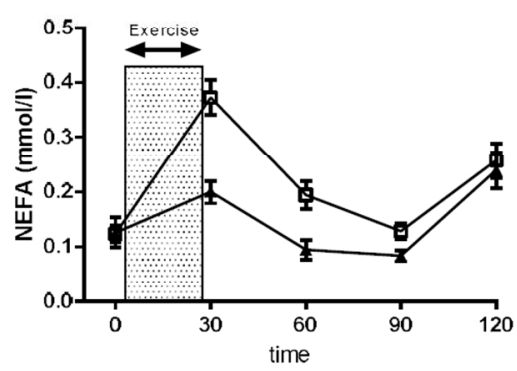

Females $(n=19)$
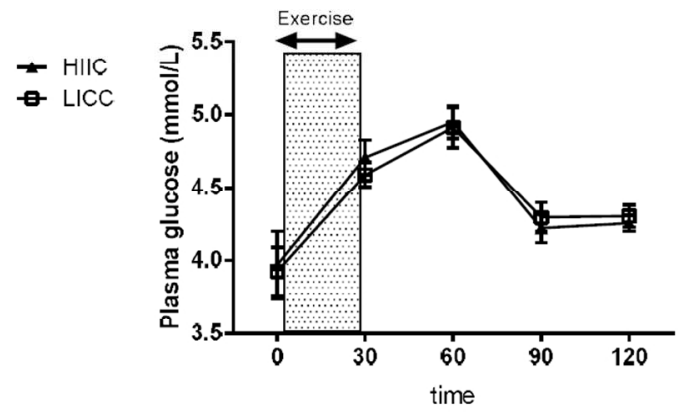

Figure 3: A. Plasma NEFA profiles pre and post LICC $(\leq)$ and HIIC $(\pi)$ exercise for males $(n=14)$. Repeated measures ANOVA showed significant effect of time $(P<0.001)$ and exercise intervention $(P=0.012)$, with significant time*exercise interaction $(P<0.001)$. B. Plasma NEFA profiles pre and post LICC $(\leq)$ and HIIC $(\pi)$ exercise for females $(n=19)$. Repeated measures ANOVA showed significant effect of time $(P<0.001)$ and exercise intervention $(P=0.013)$, with significant time*exercise interaction $(P<0.001)$. C. Plasma glucose profiles pre and post LICC $(\leq)$ and HIIC $(\pi)$ exercise for males $(n=21)$. Repeated measures ANOVA showed significant effect of time $(P<0.001)$ but no effect of exercise intervention $(P=0.142)$, however the iAUC for glucose was significantly greater post-HIIC exercise $(P<0.001)$. C. Plasma glucose profiles pre and post LICC $(\leq)$ and HIIC $(\pi)$ exercise for females $(n=19)$. Repeated measures ANOVA showed significant effect of time $(P<0.001)$ but no effect of exercise intervention $(P=0.847)$, in addition, iAUC for glucose post-exercise was not significantly different between exercise intensities $(P \geq 0.501)$.

$107 \times 84 \mathrm{~mm}(300 \times 300$ DPI $)$ 
A

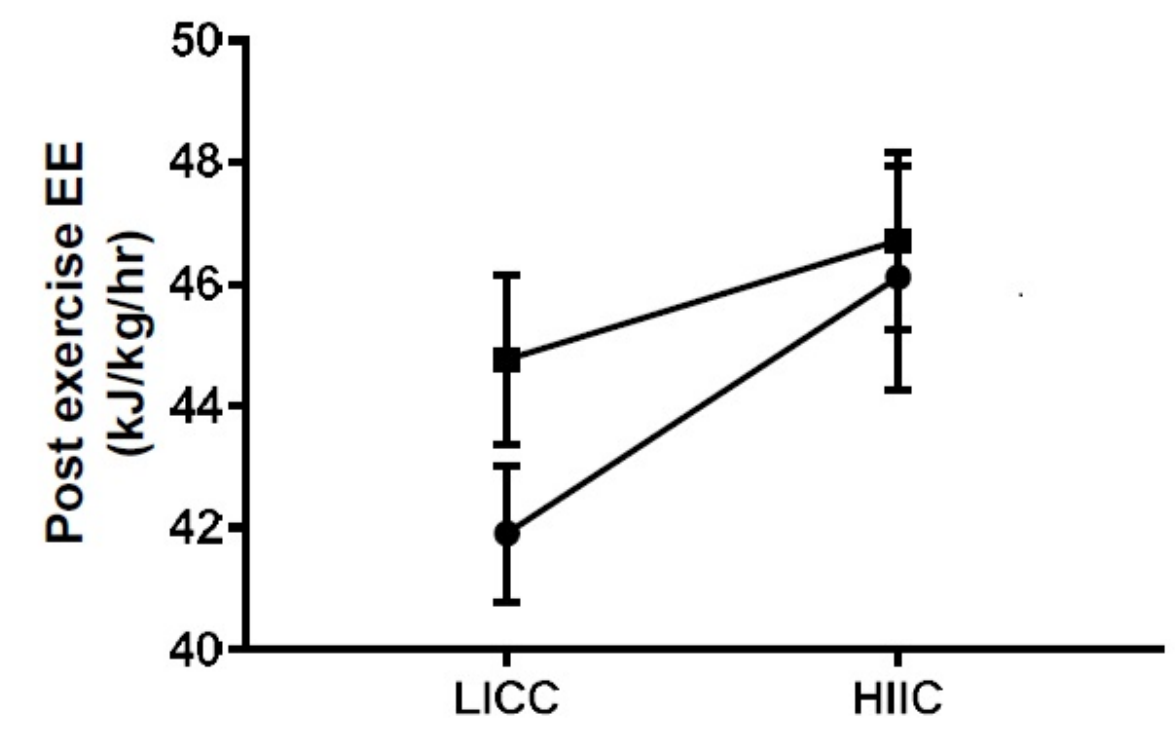

B

$\rightarrow$ Females

$\rightarrow$ Males

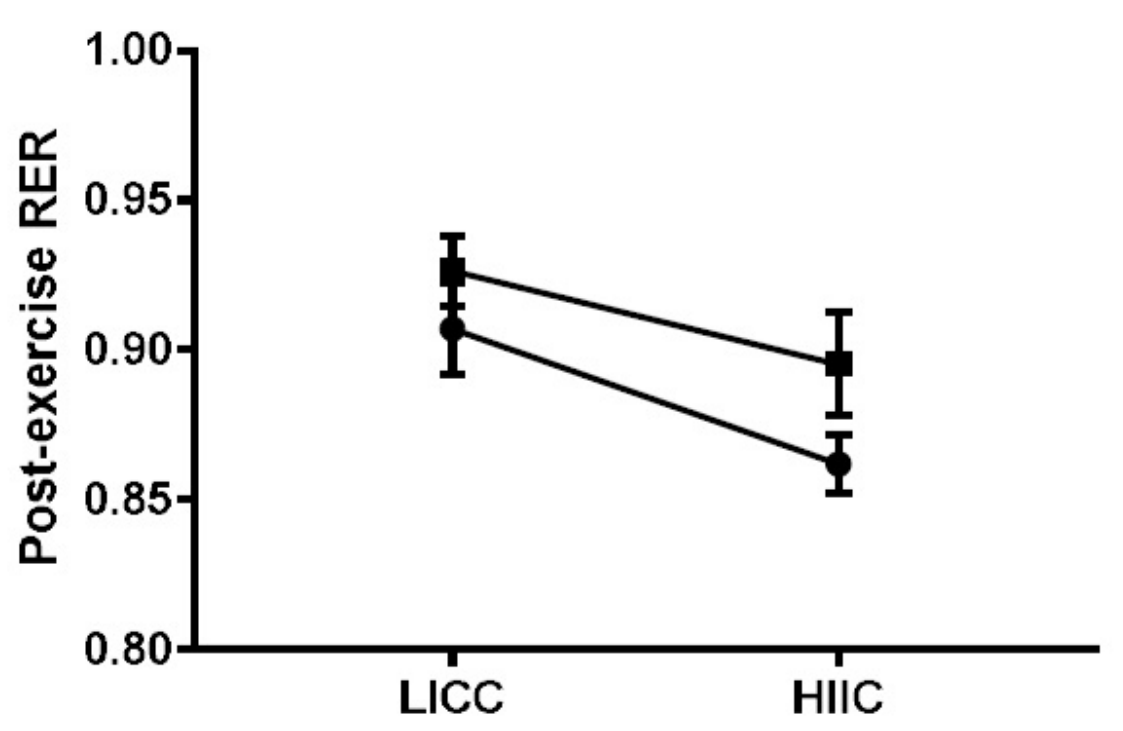

